\title{
A SPECTRAL MAPPING THEOREM FOR SEMIGROUPS SOLVING PDES WITH NONAUTONOMOUS PAST
}

\author{
GENNI FRAGNELLI
}

Received 20 June 2002

We prove a spectral mapping theorem for semigroups solving partial differential equations with nonautonomous past. This theorem is then used to give spectral conditions for the stability of the solutions of the equations.

\section{Introduction}

The aim of this paper is to prove a spectral mapping theorem for semigroups solving partial differential equations of the form

$$
\begin{gathered}
\dot{u}(t)=B u(t)+\Phi \tilde{u}_{t}, \quad t \geq 0, \\
u(0)=x \in X, \\
\tilde{u}_{0}=f \in L^{p}\left(\mathbb{R}_{-}, X\right)
\end{gathered}
$$

on some Banach space $X$, where $(B, D(B))$ is the generator of a strongly continuous semigroup $(S(t))_{t \geq 0}$ on $X$, the delay operator $\Phi$ is defined in $D(\Phi) \subseteq$ $L^{p}\left(\mathbb{R}_{-}, X\right)$ with values in $X$, and the modified history function $\tilde{u}_{t}: \mathbb{R}_{-} \rightarrow X$ is

$$
\tilde{u}_{t}(\tau):= \begin{cases}U(\tau, t+\tau) f(t+\tau), & \text { for } 0 \geq t+\tau \geq \tau, \\ U(\tau, 0) u(t+\tau), & \text { for } t+\tau \geq 0 \geq \tau,\end{cases}
$$

for an evolution family $(U(t, s))_{t \leq s \leq 0}$ on $X$. We refer to $[5,12]$ where these equations have been introduced and to $[10,13]$ for concrete examples. In particular, we showed in [12] that (1.1) and the abstract Cauchy problem associated to an operator $(\mathscr{C}, D(\mathscr{C}))$ on the product space $\mathscr{E}:=X \times L^{p}\left(\mathbb{R}_{-}, X\right)$ are "equivalent." Since, under appropriate conditions, the operator $(\mathscr{C}, D(\mathscr{C}))$ is the generator of a strongly continuous semigroup $(\mathscr{T}(t))_{t \geq 0}$ on $\mathscr{E}$, the solutions of $(1.1)$ can be obtained from this semigroup (see [12, Theorem 3.5]). 
By proving a spectral mapping theorem for this solution semigroup, we are now able to discuss the stability of the solutions of (1.1). To do this, we use the critical spectrum and the critical growth bound of a semigroup (see $[6,15])$.

The structure of the paper is the following. In Section 2, we briefly review the concepts needed for a semigroup treatment of (1.1) and recall some notations from [12]. In Section 3, we prove a spectral mapping theorem for the semigroup generated by $(\mathscr{C}, D(\mathscr{C}))$ and, in particular, we prove that, under appropriate assumptions on the operator $B$, its spectrum can be obtained from the spectrum of $\mathscr{C}$ and the spectrum of the evolution semigroup associated to the evolution family $(U(t, s))_{t \leq s \leq 0}$. In Section 4 , as an application of the spectral mapping theorem, we study the stability of the solution semigroup $(\mathscr{T}(t))_{t \geq 0}$ and discuss a concrete example.

\section{Technical preliminaries}

2.1. A semigroup approach to partial differential equations with nonautonomous delay. Since this paper is a continuation of [12], we refer to it throughout the following.

Let $(U(t, s))_{t \leq s \leq 0}$ be an (exponentially bounded, backward) evolution family (see [12, Definition 2.1]). To use semigroup techniques we extend $(U(t, s))_{t \leq s \leq 0}$ to an evolution family $(\tilde{U}(t, s))_{t \leq s}$ on $\mathbb{R}$ in a trivial way (see [12, Definition 2.2.1]). On the space $\widetilde{E}:=L^{p}(\mathbb{R}, X)$, we define the corresponding evolution semigroup $(\tilde{T}(t))_{t \geq 0}$ by

$$
(\tilde{T}(t) \tilde{f})(s):=\tilde{U}(s, s+t) \tilde{f}(s+t)
$$

for all $\tilde{f} \in \tilde{E}, s \in \mathbb{R}$, and $t \geq 0$ (see also $[2,16]$ ).

We denote its generator by $(\widetilde{G}, D(\widetilde{G}))$. Since $(\widetilde{G}, D(\widetilde{G}))$ is a local operator (see $\left[17\right.$, Theorem 2.4]), we can restrict it to the space $E:=L^{p}\left(\mathbb{R}_{-}, X\right)$ by the following definition.

Definition 2.1. Take

$$
D(G):=\left\{\tilde{f}_{\mid \mathbb{R}_{-}}: \tilde{f} \in D(\tilde{G})\right\}
$$

and define

$$
G f:=(\tilde{G} \tilde{f})_{\mid \mathbb{R}_{-}} \text {for } f=\tilde{f}_{\mid \mathbb{R}_{-}} \in D(\tilde{G})
$$

Using the operator $(G, D(G))$, we can define a new operator.

Definition 2.2. Let $\mathscr{C}$ be the operator defined as

$$
\mathscr{C}:=\left(\begin{array}{ll}
B & \Phi \\
0 & G
\end{array}\right),
$$


with domain

$$
D(\mathscr{b}):=\left\{\left(\begin{array}{l}
x \\
f
\end{array}\right) \in D(B) \times D(G): f(0)=x\right\},
$$

on the product space $\mathscr{E}:=X \times L^{p}\left(\mathbb{R}_{-}, X\right)$.

In [12] we reformulated (1.1), given in the introduction, as the abstract Cauchy problem

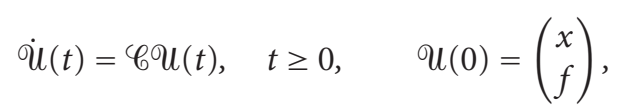

on $\mathscr{E}:=X \times L^{p}\left(\mathbb{R}_{-}, X\right)$ and we proved the following result relating (1.1) to the abstract Cauchy problem with the operator $(\mathscr{C}, D(\mathscr{C}))$.

Theorem 2.3. The delay equation with nonautonomous past (1.1) is well posed if and only if the operator $(\mathscr{C}, D(\mathscr{C}))$ is the generator of a strongly continuous semigroup $\mathscr{T}:=(\mathscr{T}(t))_{t \geq 0}$ on $\mathscr{E}$.

In that case, (1.1) has a unique solution u for every $\left(\begin{array}{l}x \\ f\end{array}\right) \in D(\mathscr{C})$, given by

$$
u(t):= \begin{cases}\pi_{1}\left(\mathcal{T}(t)\left(\begin{array}{l}
x \\
f
\end{array}\right)\right), & t \geq 0 \\
f(t), & t \leq 0\end{cases}
$$

where $\pi_{1}$ is the projection onto the first component of $\mathscr{E}$.

From now on we make the following assumption for the delay operator $\Phi$.

Assumption 2.4. Let $1<p<\infty$ and let $\eta: \mathbb{R}_{-} \rightarrow \mathscr{L}(X)$ be of bounded variation such that $|\eta|\left(\mathbb{R}_{-}\right)<+\infty$, where $|\eta|$ is the positive Borel measure in $\mathbb{R}_{-}$defined by the total variation on $\eta$. Assume that the linear delay operator $\Phi$ in (1.1) has the representation given by the following Riemann-Stieltjes integral:

$$
\Phi f:=\int_{-\infty}^{0} f d \eta \quad \forall f \in C_{0}\left(\mathbb{R}_{-}, X\right) \cap L^{p}\left(\mathbb{R}_{-}, X\right),
$$

which is well defined by [1, Proposition 1.9.4].

In particular, as in [3, Chapter I.2], we can take

$$
\Phi f:=\int_{-\infty}^{0} \phi(s) f(s) d s
$$

where $\phi(\cdot) \in L^{1}\left(\mathbb{R}_{-}\right)$, or

$$
\Phi f:=\delta_{s} f
$$

where $\delta_{s}$ is the Dirac measure for some $s<0$. 
The next result is needed to state the most important result of this section. Let $\mathscr{C}_{0}$ be the operator

$$
\mathscr{C}_{0}:=\left(\begin{array}{cc}
B & 0 \\
0 & G
\end{array}\right)
$$

with domain $D\left(\mathscr{C}_{0}\right)=D(\mathscr{C})$, where $G$ is the operator defined in Definition 2.1.

Proposition 2.5 (see [12, Proposition 4.2]). The operator $\left(\mathscr{C}_{0}, D\left(\mathscr{C}_{0}\right)\right)$ generates the strongly continuous semigroup $\left(\mathscr{T}_{0}(t)\right)_{t \geq 0}$ on $\mathscr{E}$ given by

$$
\mathscr{T}_{0}(t):=\left(\begin{array}{cc}
S(t) & 0 \\
S_{t} & T_{0}(t)
\end{array}\right),
$$

where $\left(T_{0}(t)\right)_{t \geq 0}$ is the evolution semigroup on $E$ associated to the evolution family $(U(t, s))_{t \leq s \leq 0}$, that is,

$$
\left(T_{0}(t) f\right)(s):= \begin{cases}U(s, s+t) f(t+s), & s+t \leq 0 \\ 0, & s+t>0\end{cases}
$$

for $f \in E$ and $S_{t}: X \rightarrow E$ is

$$
\left(S_{t} x\right)(s):= \begin{cases}U(s, 0) S(t+s) x, & s+t>0 \\ 0, & s+t \leq 0\end{cases}
$$

Recall that $(S(t))_{t \geq 0}$ is the semigroup generated by the operator $(B, D(B))$.

For the delay operator $\Phi$ as in Assumption 2.4 the following theorem holds.

Theorem 2.6. Let $\Phi$ be as in Assumption 2.4. Then the operator $(\mathscr{C}, D(\mathscr{C}))$ generates a strongly continuous semigroup $(\mathcal{T}(t))_{t \geq 0}$ on $\mathscr{E}$ given by the Dyson-Phillips series

$$
\mathscr{T}(t)=\sum_{n=0}^{+\infty} \mathcal{T}_{n}(t)
$$

for all $t \geq 0$, where $\left(\mathscr{T}_{0}(t)\right)_{t \geq 0}$ is the semigroup given by (2.12) and

$$
\mathscr{T}_{n+1}(t)\left(\begin{array}{l}
x \\
f
\end{array}\right):=\int_{0}^{t} \mathcal{T}_{0}(t-s) \mathscr{F}_{F} \mathcal{T}_{n}(s)\left(\begin{array}{l}
x \\
f
\end{array}\right) d s
$$

for each $\left(\begin{array}{l}x \\ f\end{array}\right) \in D(\mathscr{b}), t \geq 0$, and $\mathscr{F}$ is the operator matrix

$$
\left(\begin{array}{ll}
0 & \Phi \\
0 & 0
\end{array}\right)
$$

such that $\mathscr{F} \in \mathscr{L}\left(D\left(\mathscr{C}_{0}\right), \mathscr{E}\right)$. In particular (1.1) is well posed. 
The proof of this theorem is based on the perturbation theorem of MiyaderaVoigt (see [8, Theorem III.3.14] and [14, 20]).

THEOREM 2.7. Let $\left(W_{0}(t)\right)_{t \geq 0}$ be a strongly continuous semigroup on the Banach space $X$ with generator $\left(H_{0}, D\left(H_{0}\right)\right)$. Let $F: D\left(H_{0}\right) \rightarrow X$ be a linear operator on $X$ such that there exists a function $q: \mathbb{R}_{+} \rightarrow \mathbb{R}_{+}$satisfying $\lim _{t \rightarrow 0} q(t)=0$ and

$$
\int_{0}^{t}\left\|F W_{0}(t) x\right\| d t \leq q(t)\|x\|
$$

for all $x \in D\left(H_{0}\right)$ and $t>0$.

Then $\left(H_{0}+F, D\left(H_{0}\right)\right)$ generates a strongly continuous semigroup $(W(t))_{t \geq 0}$ on $\mathscr{E}$ given by the Dyson-Phillips series

$$
W(t)=\sum_{n=0}^{+\infty} W_{n}(t)
$$

for all $t \geq 0$, where

$$
W_{n+1}(t) x:=\int_{0}^{t} W_{0}(t-s) F W_{n}(s) x d s
$$

for all $x \in D\left(H_{0}\right), t \geq 0$.

Proof of Theorem 2.6. Taking in Theorem 2.7 the semigroup $\left(\mathscr{T}_{0}(t)\right)_{t \geq 0}$ as $\left(W_{0}(t)\right)_{t \geq 0}$, the operator $\mathscr{C}_{0}$ as $H_{0}$, and the operator $\mathscr{F}$ as $F$, then we have

$$
\begin{aligned}
\left\|\mathscr{F}_{\mathcal{F}_{0}(t)}\left(\begin{array}{l}
x \\
f
\end{array}\right)\right\| & =\left\|\left(\begin{array}{cc}
0 & \Phi \\
0 & 0
\end{array}\right)\left(\begin{array}{cc}
S(t) & 0 \\
S_{t} & T_{0}(t)
\end{array}\right)\left(\begin{array}{l}
x \\
f
\end{array}\right)\right\| \\
& =\left\|\left(\begin{array}{c}
\Phi\left(S_{t} x+T_{0}(t) f\right) \\
0
\end{array}\right)\right\| \\
& =\left\|\Phi\left(S_{t} x+T_{0}(t) f\right)\right\|
\end{aligned}
$$

for all $\left(\begin{array}{l}x \\ f\end{array}\right) \in D\left(\mathscr{C}_{0}\right)$. It follows that condition (2.18) is equivalent to

$$
\int_{0}^{t}\left\|\Phi\left(S_{r} x+T_{0}(r) f\right)\right\| d r \leq q(t)\left\|\left(\begin{array}{l}
x \\
f
\end{array}\right)\right\|
$$

for $t>0$ and each $\left(\begin{array}{l}x \\ f\end{array}\right) \in D\left(\mathscr{C}_{0}\right)$.

Moreover, as in [12, Example 4.6], we can prove that the delay operator $\Phi$ given in Assumption 2.4 satisfies (2.22) for $q(t)=M M_{\omega} e^{|\omega| t}|\eta|\left(\mathbb{R}_{-}\right) t^{1 / p^{\prime}}$, where $M:=\sup _{r \in[0,1]}\|S(r)\|$ and $M_{\omega}, \omega$, and $p^{\prime}$ are such that $\|U(t, s)\| \leq M_{\omega} e^{\omega(s-t)}$ for $t \leq s \leq 0$ and $(1 / p)+\left(1 / p^{\prime}\right)=1$, respectively. Hence, we can apply Theorem 2.7 and the proof is complete. 
Remark 2.8. The operator $\mathscr{C}$ given in Definition 2.2 is a perturbation of $\mathscr{C}_{0}$ given in (2.11), hence the solution semigroup $(\mathscr{T}(t))_{t \geq 0}$ is a perturbation of the semigroup $\left(\mathscr{T}_{0}(t)\right)_{t \geq 0}$.

2.2. The critical spectrum and the critical growth bound. Nagel and Poland introduced in [15] the critical spectrum and proved a corresponding spectral mapping theorem (see also $[4,6]$ ). We briefly recall its definitions.

Let $X$ be a Banach space and $\mathcal{T}:=(T(t))_{t \geq 0}$ a strongly continuous semigroup on $X$. We can extend this semigroup to a semigroup $\tilde{\mathscr{T}}:=(\tilde{T}(t))_{t \geq 0}$ on $\tilde{X}:=$ $\left\{\left(x_{n}\right)_{n \in \mathbb{N}} \subset X: \sup _{n \in \mathbb{N}}\left\|x_{n}\right\|<\infty\right\}$, no longer strongly continuous, by

$$
\widetilde{T}(t)\left(x_{n}\right):=\left(T(t) x_{n}\right)_{n \in \mathbb{N}}, \quad\left(x_{n}\right)_{n \in \mathbb{N}} \in \tilde{X}
$$

Now, the subspace

$$
\tilde{X}_{\mathscr{T}}:=\left\{\left(x_{n}\right)_{n \in \mathbb{N}} \in \tilde{X}: \limsup _{t \downarrow 0}\left\|T(t) x_{n}-x_{n}\right\|=0\right\}
$$

is closed and $(\widetilde{T}(t))_{t \geq 0}$-invariant. Therefore, the quotient operators

$$
\hat{T}(t)\left(\tilde{x}+\tilde{X}_{\mathscr{T}}\right):=\tilde{T}(t) \tilde{x}+\tilde{X}_{\mathscr{T}}, \quad \tilde{x}+\tilde{X}_{\mathscr{T}} \in \hat{X},
$$

are well defined on the quotient space

$$
\hat{X}:=\tilde{X} / \tilde{X}_{\mathscr{T}}
$$

and yield a semigroup $\hat{\mathscr{T}}:=(\hat{T}(t))_{t \geq 0}$ of bounded operators on $\hat{X}$.

Definition 2.9. The critical spectrum of the semigroup $(T(t))_{t \geq 0}$ is defined as

$$
\sigma_{\text {crit }}(T(t)):=\sigma(\hat{T}(t)), \quad t \geq 0
$$

while the critical growth bound is

$$
\omega_{\text {crit }}(\mathscr{T}(t)):=\inf \left\{\omega \in \mathbb{R}: \exists M \geq 1 \text { such that }\|\hat{T}(t)\| \leq M e^{\omega t} \forall t \geq 0\right\} .
$$

We now determine the critical spectrum of a special class of semigroups. This result will be useful to prove the spectral mapping theorem for the semigroup obtained in Theorem 2.6.

As a first step, it can be shown as in [7, Theorem 3.22], [8, Section VI.9], [18, Theorem 2.3], or [19, Corollary 2.4] that the spectral mapping theorem holds for the semigroup $\left(T_{0}(t)\right)_{t \geq 0}$ given in (2.13). 
Theorem 2.10. Let $\left(T_{0}(t)\right)_{t \geq 0}$ be the semigroup on E given in (2.13) and $\left(G_{0}\right.$, $\left.D\left(G_{0}\right)\right)$ its generator. Then $\sigma\left(T_{0}(t)\right)$ is a disk centered at the origin and the spectrum $\sigma\left(G_{0}\right)$ is a half-plane. Moreover, $\left(T_{0}(t)\right)_{t \geq 0}$ satisfies the spectral mapping theorem

$$
\sigma\left(T_{0}(t)\right) \backslash\{0\}=e^{t \sigma\left(G_{0}\right)}, \quad t \geq 0
$$

In particular, $s\left(G_{0}\right)=\omega_{0}\left(T_{0}(\cdot)\right)=\omega_{0}(\mathcal{U})$, where $s\left(G_{0}\right)$ is the spectral bound of $G_{0}$, and $\omega_{0}\left(T_{0}(\cdot)\right)$ and $\omega_{0}(\mathcal{U})$ are the growth bounds of $\left(T_{0}(t)\right)_{t \geq 0}$ and $(U(t, s))_{t \leq s \leq 0}$, respectively.

It turns out that in this case spectrum and critical spectrum coincide.

Theorem 2.11. The critical spectrum of $T_{0}:=\left(T_{0}(t)\right)_{t \geq 0}$ coincides with its spectrum, that is,

$$
\sigma_{\text {crit }}\left(T_{0}(t)\right)=\sigma\left(T_{0}(t)\right), \quad t \geq 0
$$

Proof. We only have to prove the inclusion $\sigma_{\text {crit }}\left(T_{0}(t)\right) \supseteq \sigma\left(T_{0}(t)\right)$ for $t \geq 0$.

Using rescaling, as in [15], the inclusion follows if we can show that

$$
2 \pi i \mathbb{Z} \in \sigma\left(G_{0}\right) \Longrightarrow 1 \in \sigma_{\text {crit }}\left(T_{0}(1)\right) \text {. }
$$

Since the spectrum $\sigma\left(G_{0}\right)$ is the union of the approximate point spectrum $A \sigma\left(G_{0}\right)$ and the residual spectrum $R \sigma\left(G_{0}\right)$ (see, e.g., [8, Section IV.1]), it follows from $2 \pi i \mathbb{Z} \subset \sigma\left(G_{0}\right)$ that at least one of the sets

$$
A \sigma\left(G_{0}\right) \cap 2 \pi i \mathbb{Z} \quad \text { or } \quad R \sigma\left(G_{0}\right) \cap 2 \pi i \mathbb{Z}
$$

is unbounded. In the first case the assertion follows from [4, Proposition 4]. Assume now that $2 \pi i k_{n} \in R \sigma\left(G_{0}\right)$ for some unbounded sequence $\left(k_{n}\right)_{n \in \mathbb{N}}$.

Observe now (see [8, Proposition IV.2.18]) that

$$
\sigma_{\text {crit }}\left(T_{0}(t)\right)=\sigma\left(\hat{T}_{0}(t)\right)=\sigma\left(\hat{T}_{0}^{\prime}(t)\right)
$$

on

$$
(\hat{X})^{\prime}=\left(\tilde{X} / \tilde{X}_{T_{0}}\right)^{\prime} \cong\left(\tilde{X}_{T_{0}}\right)^{\circ} \subset(\tilde{X})^{\prime}
$$

where $\left(\tilde{X}_{T_{0}}\right)^{\circ}$ is the dual of $\tilde{X}_{T_{0}}$ (see [8, Definition II.2.6]). By [8, Proposition IV.2.18], one has

$$
2 \pi i k_{n} \in R \sigma\left(G_{0}\right)=P \sigma\left(G_{0}^{\prime}\right) \quad \forall n \in \mathbb{N} .
$$

Therefore, there exists $x_{n}^{\prime} \in X^{\prime},\left\|x_{n}^{\prime}\right\|=1$, such that $T_{0}^{\prime}(t) x_{n}^{\prime}=e^{2 \pi i k_{n} t} x_{n}^{\prime}$ for $t \geq 0$ and all $n \in \mathbb{N}$. 
We define

$$
y_{n}^{\prime}:=x_{n}^{\prime}-T_{0}^{\prime}\left(\frac{1}{2 k_{n}}\right) x_{n}^{\prime}=2 x_{n}^{\prime} \quad \text { for } n \in \mathbb{N} .
$$

It holds that $T_{0}^{\prime}(1) y_{n}^{\prime}=y_{n}^{\prime}$ and

$$
\lim _{n \rightarrow \infty}\left\langle y_{n}^{\prime}, x_{n}\right\rangle=\lim _{n \rightarrow \infty}\left\langle x_{n}^{\prime}, x_{n}-T_{0}\left(\frac{1}{2 k_{n}}\right) x_{n}\right\rangle=0
$$

for all $\left(x_{n}\right)_{n \in \mathbb{N}} \in \tilde{X}_{T_{0}}$.

Define $y^{\prime} \in(\tilde{X})^{\prime}$ so that

$$
\left\langle y^{\prime},\left(x_{n}\right)\right\rangle:=\psi\left(\left\langle y_{n}^{\prime}, x_{n}\right\rangle\right) \quad \forall\left(x_{n}\right) \in \tilde{X}
$$

where $\psi$ is a Banach limit on $l^{\infty}$ (see [9, Chapter V.0]). By (2.37),

$$
y^{\prime} \in\left(\tilde{X}_{T_{0}}\right)^{\circ}, \quad \widetilde{T}_{0}(1)^{\prime} y^{\prime}=\left(T_{0}^{\prime}(1) y^{\prime}\right)=y^{\prime} .
$$

Thus $\hat{T}_{0}^{\prime}(1) y^{\prime}=y^{\prime}$ and $1 \in \sigma\left(\hat{T}_{0}(1)\right)$.

Corollary 2.12. The critical growth bound of the evolution semigroup $\left(T_{0}(t)\right)_{t \geq 0}$ is equal to the growth bound of the corresponding evolution family $U:=(U(t$, $s))_{t \leq s \leq 0}$, that is,

$$
\omega_{\text {crit }}\left(T_{0}(\cdot)\right)=\omega_{0}(\mathcal{U})=\omega_{0}\left(T_{0}(\cdot)\right)
$$

Proof. By Theorem 2.11, we have

$$
\omega_{\text {crit }}\left(T_{0}(\cdot)\right)=\omega_{0}\left(T_{0}(\cdot)\right) \text {, }
$$

while Theorem 2.10 implies

$$
\omega_{0}\left(T_{0}(\cdot)\right)=\omega_{0}(\mathcal{U})
$$

\section{The spectral mapping theorem}

In this section, we want to prove a spectral mapping theorem for the semigroup $(\mathscr{T}(t))_{t \geq 0}$ from Section 2. First, we determine the spectrum $\sigma(\mathscr{C})$ of the operator $\mathscr{C}$ by some analogue of the characteristic equation for delay equations (see [8, Chapter VI]).

Lemma 3.1 (see [12, Lemma 5.1]). For $\lambda \in \mathbb{C}$ with $\mathfrak{R} \lambda>\omega_{0}(\boldsymbol{U})$,

$$
\lambda \in \sigma(\mathscr{C}) \quad \text { iff } \lambda \in \sigma\left(B+\Phi \epsilon_{\lambda}\right),
$$


where the bounded operator $\epsilon_{\lambda}: X \rightarrow E$ is defined by

$$
\left(\epsilon_{\lambda} x\right)(s):=e^{\lambda s} U(s, 0) x, \quad s \leq 0, x \in X
$$

In order to obtain our spectral mapping theorem, we need an assumption on the semigroup without delay, that is, on the semigroup $(S(t))_{t \geq 0}$ generated by $B$. The following turns out to be appropriate.

Assumption 3.2. The operator $(B, D(B))$ generates an immediately norm continuous semigroup $(S(t))_{t \geq 0}$ on $X$, that is, the function

$$
t \longmapsto S(t) \in \mathscr{L}(X)
$$

is norm continuous for all $t>0$ (see [8, Definition II.4.17 or Theorem IV.3.10]).

Analytic semigroups are typical examples of immediately norm continuous semigroups.

Define now

$$
V(t):=\left(\begin{array}{cc}
S(t) & 0 \\
0 & T_{0}(t)
\end{array}\right), \quad Q(t):=\left(\begin{array}{cc}
0 & 0 \\
-S_{t} & 0
\end{array}\right)
$$

for $t \geq 0$, where $S_{t}$ is defined as in (2.14).

Remark 3.3. It is easy to prove that $(V(t))_{t \geq 0}$ is a semigroup on the product space $\mathscr{E}$. Moreover, by the definitions of $V(t)$ and $Q(t)$,

$$
V(t)=\mathscr{T}_{0}(t)+Q(t) \quad \forall t \geq 0,
$$

where $\left(\mathscr{T}_{0}(t)\right)_{t \geq 0}$ is the semigroup given in Proposition 2.5.

In the next step, we extend, in the canonical way, both semigroups $\left(\mathscr{T}_{0}(t)\right)_{t \geq 0}$ and $(V(t))_{t \geq 0}$ to semigroups $\widetilde{\mathscr{T}}_{0}:=\left(\widetilde{\mathscr{T}}_{0}(t)\right)_{t \geq 0}$ and $\tilde{V}:=(\tilde{V}(t))_{t \geq 0}$ on $\widetilde{\mathscr{E}}=l^{\infty}(\mathscr{E})$ (see Section 2.2 for the definition) and show that their spaces of strong continuity coincide.

LEMMA 3.4. It results that $\stackrel{\widetilde{\mathscr{E}}}{\widetilde{V}}_{\tilde{\mathscr{E}}}=\widetilde{\mathscr{G}}_{\widetilde{T}_{0}}$.

Proof. Using the definitions of $(V(t))_{t \geq 0}$ and $\left(\mathscr{T}_{0}(t)\right)_{t \geq 0}$, we obtain

$$
\begin{aligned}
\left\|V(h)\left(\begin{array}{l}
x \\
f
\end{array}\right)-\mathscr{T}_{0}(h)\left(\begin{array}{l}
x \\
f
\end{array}\right)\right\| & =\left\|\left(\begin{array}{c}
S(h) x-S(h) x \\
T_{0}(h) f-S_{h} x-T_{0}(h) f
\end{array}\right)\right\| \\
& =\left\|\left(\begin{array}{c}
0 \\
-S_{h} x
\end{array}\right)\right\| \leq\left\|S_{h} x\right\| .
\end{aligned}
$$


942 SMT for semigroups solving PDEs with nonautonomous past

However,

$$
\begin{aligned}
\left\|S_{h} x\right\|_{L^{p}\left(\mathbb{R}_{-}, X\right)}^{p} & =\int_{\mathbb{R}_{-}}\left\|\left(S_{h} x\right)(\tau)\right\|^{p} d \tau \\
& =\int_{-h}^{0}\|U(\tau, 0) S(h+\tau) x\|^{p} d \tau \\
& \leq \int_{-h}^{0} M_{\omega}^{p} e^{-\omega \tau p} M_{\bar{\omega}}^{p} e^{\bar{\omega}(h+\tau) p}\|x\|^{p} d \tau \\
& \leq C^{p} e^{p h(|\omega|+|\bar{\omega}|)} h\|x\|^{p},
\end{aligned}
$$

where $\omega, M_{\omega}$ and $\bar{\omega}, M_{\bar{\omega}}$ are such that $\|U(t, s)\| \leq M_{\omega} e^{\omega(s-t)}$ for $t \leq s \leq 0$ and $\|S(t)\| \leq M_{\bar{\omega}} e^{t \bar{\omega}}$ for $t \geq 0$, respectively, and $C:=M_{\omega} M_{\bar{\omega}}$.

Clearly, the last term in (3.7) goes to zero as $h \backslash 0$.

The following two lemmas will be used in Proposition 3.7. In particular, Lemma 3.5 is important to prove the norm continuity of $Q(t)$.

Lemma 3.5. The function $[0,+\infty) \ni t \mapsto S_{t} \in \mathscr{L}(X, E)$ is norm continuous.

Proof. Let $t \geq 0, M:=\sup _{t \in[0,1]}\|S(t)\|$, and $1>h>0$. One has

$$
\begin{aligned}
& \lim _{h \rightarrow 0^{+}}\left\|S_{t+h}-S_{t}\right\| \\
& =\lim _{h \rightarrow 0^{+}} \sup _{\|x\| \leq 1}\left(\int_{\mathbb{R}_{-}}\left\|\left(S_{t+h} x\right) \tau-\left(S_{t} x\right) \tau\right\|_{X}^{p} d \tau\right)^{1 / p} \\
& =\lim _{h \rightarrow 0^{+}\|x\| \leq 1} \sup _{\| x}\left(\int_{-(t+h)}^{-t}\|U(\tau, 0) S(t+h+\tau) x\|^{p} d \tau\right. \\
& \left.\quad+\int_{-t}^{0}\|U(\tau, 0) S(t+h+\tau) x-U(\tau, 0) S(t+\tau) x\|^{p} d \tau\right)^{1 / p} \\
& \leq \lim _{h \rightarrow 0^{+}\|x\| \leq 1}\left(\int_{-(t+h)}^{-t}\left(M_{\omega} e^{-\omega \tau}\|S(t+h+\tau)\|\|x\|\right)^{p} d \tau\right. \\
& \left.\quad+\|x\|^{p} \int_{-t}^{0}\|U(\tau, 0)\|^{p}\|S(t+h+\tau)-S(t+\tau)\|^{p} d \tau\right)^{1 / p} \\
& \leq \lim _{h \rightarrow 0^{+}\|x\| \leq 1} \sup _{\omega}\|x\| e^{|\omega|(t+h)}\left(\int_{-(t+h)}^{-t}\|S(t+h+\tau)\|^{p} d \tau\right)^{1 / p} \\
& \quad+\lim _{h \rightarrow 0^{+}\|x\| \leq 1} M_{\omega}\|x\|\left(\int_{-t}^{0} e^{-\omega \tau p}\|S(t+h+\tau)-S(t+\tau)\|^{p} d \tau\right)^{1 / p}
\end{aligned}
$$




$$
\begin{aligned}
\leq & \lim _{h \rightarrow 0^{+}} M_{\omega} e^{|\omega|(t+h)}\left(\int_{0}^{h}\|S(\sigma)\|^{p} d \sigma\right)^{1 / p} \\
& +\lim _{h \rightarrow 0^{+}} M_{\omega} e^{|\omega| t}\left(\int_{-t}^{0}\|S(t+h+\tau)-S(t+\tau)\|^{p} d \tau\right)^{1 / p} \\
\leq & \lim _{h \rightarrow 0^{+}} M_{\omega} e^{|\omega|(t+h)} M h \\
& +\lim _{h \rightarrow 0^{+}} M_{\omega} e^{|\omega| t}\left(\int_{-t}^{0}\|S(t+h+\tau)-S(t+\tau)\|^{p} d \tau\right)^{1 / p} .
\end{aligned}
$$

The last term goes to zero as $h \rightarrow 0^{+}$since $(S(t))_{t \geq 0}$ is immediately norm continuous by Assumption 3.2 and therefore uniformly norm continuous on compact intervals.

The proof for $h \rightarrow 0^{-}$is similar.

As a consequence of Lemma 3.5, we obtain that the function $t \mapsto Q(t)$ is norm continuous from $[0,+\infty)$ to $\mathscr{L}(\mathscr{E})$.

The next lemma relates the semigroup $\left(\mathcal{T}_{0}(t)\right)_{t \geq 0}$ and the operators $Q(t)$.

Lemma 3.6. With the definitions above,

$$
\lim _{h \downarrow 0}\left\|\mathscr{T}_{0}(h) Q(t)-Q(t+h)\right\|=0 \quad \forall t \geq 0
$$

Proof. Using the definitions of $\left(\mathscr{T}_{0}(t)\right)_{t \geq 0}$ and $Q(t)$, we have

$$
\begin{aligned}
& \left\|\mathscr{T}_{0}(h) Q(t)-Q(t+h)\right\| \\
& =\sup _{\left\|\left(\begin{array}{l}
x \\
f
\end{array}\right)\right\| \leq 1}\left\|\left(\begin{array}{cc}
S(h) & 0 \\
S_{h} & T_{0}(h)
\end{array}\right)\left(\begin{array}{cc}
0 & 0 \\
-S_{t} & 0
\end{array}\right)\left(\begin{array}{l}
x \\
f
\end{array}\right)-\left(\begin{array}{cc}
0 & 0 \\
-S_{t+h} & 0
\end{array}\right)\left(\begin{array}{l}
x \\
f
\end{array}\right)\right\| \\
& =\sup _{\left\|\left(\begin{array}{l}
x \\
f
\end{array}\right)\right\| \leq 1}\left\|\left(\begin{array}{c}
0 \\
-T_{0}(h) S_{t} x+S_{t+h} x
\end{array}\right)\right\| .
\end{aligned}
$$

Since, see [12, Proposition 4.2], $S_{t+h}=S_{h} S(t)+T_{0}(h) S_{t}$, we obtain

$$
\left\|\left(\begin{array}{c}
0 \\
-T_{0}(h) S_{t} x+S_{t+h} x
\end{array}\right)\right\|=\left\|\left(\begin{array}{c}
0 \\
S_{h} S(t) x
\end{array}\right)\right\|
$$

for all $x \in X$. As in Lemma 3.4 we can prove that

$$
\left\|S_{h} S(t) x\right\|_{L^{p}\left(\mathbb{R}_{-}, X\right)} \leq C e^{h|\omega|} e^{(h+t)|\bar{\omega}|} h^{1 / p}\|x\|
$$


944 SMT for semigroups solving PDEs with nonautonomous past

hence

$$
\sup _{\|x\| \leq 1}\left\|S_{h} S(t) x\right\|_{L^{p}\left(\mathbb{R}_{-}, X\right)} \leq C e^{h|\omega|} e^{(h+t)|\bar{\omega}|} h^{1 / p}
$$

Since in (3.13) the right term tends to zero as $h \searrow 0$, the proof is complete.

The following proposition gives a relation between the critical spectra of $(V(t))_{t \geq 0}$ and $\left(\mathscr{T}_{0}(t)\right)_{t \geq 0}$. In the proof we follow the idea of [6, Theorem 4.5].

Proposition 3.7. The critical spectrum of the semigroup $(V(t))_{t \geq 0}$ is equal to the critical spectrum of $\left(\mathscr{T}_{0}(t)\right)_{t \geq 0}$, that is,

$$
\sigma_{\text {crit }}(V(t))=\sigma_{\text {crit }}\left(\mathscr{T}_{0}(t)\right) \text { for } t \geq 0
$$

Proof. Using the norm continuity of $Q(t)$ and Lemma 3.6, we obtain

$$
\begin{aligned}
\lim _{h \downarrow 0}\left\|\mathcal{T}_{0}(h) Q(t)-Q(t)\right\| \\
\quad \leq \lim _{h \downarrow 0}\left(\left\|\mathscr{T}_{0}(h) Q(t)-Q(t+h)\right\|+\|Q(t+h)-Q(t)\|\right)=0
\end{aligned}
$$

for every $t \geq 0$. This implies that $\widetilde{Q}(t)$ maps $\tilde{\mathscr{E}}$ into $\tilde{\mathscr{E}}_{\mathscr{T}_{0}}$, hence $\hat{Q}(t)=0$ for $t \geq 0$.

Therefore, we have

$$
\widehat{V}(t)=\widehat{\mathscr{J}}_{0}(t)
$$

and hence

$$
\sigma_{\text {crit }}(V(t))=\sigma_{\text {crit }}\left(\mathscr{T}_{0}(t)\right) \text { for } t \geq 0
$$

We can now relate the critical spectrum of $\left(\mathscr{T}_{0}(t)\right)_{t \geq 0}$ to the critical spectrum of $\left(T_{0}(t)\right)_{t \geq 0}$.

TheOREM 3.8. The critical spectra of $\left(\mathscr{T}_{0}(t)\right)_{t \geq 0}$ on $\mathscr{E}$ and $\left(T_{0}(t)\right)_{t \geq 0}$ on $L^{p}\left(\mathbb{R}_{-}, X\right)$ coincide, that is,

$$
\sigma_{\text {crit }}\left(\mathscr{T}_{0}(t)\right)=\sigma_{\text {crit }}\left(T_{0}(t)\right) \quad \text { for } t>0
$$

Proof. By Remark 3.3, we know that

$$
V(t)=\mathscr{T}_{0}(t)+Q(t)
$$


and, using Proposition 3.7, we have

$$
\sigma_{\text {crit }}(V(t))=\sigma_{\text {crit }}\left(\mathscr{T}_{0}(t)\right)
$$

By the immediate norm continuity of $(S(t))_{t \geq 0}$ (see Assumption 3.2), we conclude that

$$
\sigma_{\text {crit }}(V(t))=\sigma_{\text {crit }}\left(T_{0}(t)\right) \cup \sigma_{\text {crit }}(S(t))=\sigma_{\text {crit }}\left(T_{0}(t)\right) \cup\{0\}=\sigma_{\text {crit }}\left(T_{0}(t)\right)
$$

Hence, the thesis follows.

Using Theorem 3.8 and Theorem 2.11, the following result is immediate.

Corollary 3.9. The critical spectrum of $\left(\mathscr{T}_{0}(t)\right)_{t \geq 0}$ is equal to the spectrum of the evolution semigroup $\left(T_{0}(t)\right)_{t \geq 0}$, that is,

$$
\sigma_{\text {crit }}\left(\mathscr{T}_{0}(t)\right)=\sigma\left(T_{0}(t)\right) \text { for } t>0
$$

The next goal is to prove that the critical spectrum of the perturbed semigroup $(\mathscr{T}(t))_{t \geq 0}$ and of the unperturbed semigroup $\left(\mathscr{T}_{0}(t)\right)_{t \geq 0}$ coincide. The basic idea for the proof of this result is to prove that the first term $\mathscr{T}_{1}(t)$ of the Dyson-Phillips series of $(\mathscr{T}(t))_{t \geq 0}$ (see Theorem 2.6) is norm continuous.

Proposition 3.10. The function

$$
t \longmapsto \mathscr{T}_{1}(t)
$$

is norm continuous for $t \geq 0$.

Proof. The first Dyson-Phillips term $\mathscr{T}_{1}(t)$ applied to $\left(\begin{array}{l}x \\ f\end{array}\right) \in D\left(\mathscr{C}_{0}\right)$ yields

$$
\begin{aligned}
\mathscr{T}_{1}(t)\left(\begin{array}{l}
x \\
f
\end{array}\right) & =\int_{0}^{t} \mathscr{T}_{0}(t-s) \mathscr{F}_{0} \mathcal{T}_{0}(s)\left(\begin{array}{l}
x \\
f
\end{array}\right) d s \\
& =\int_{0}^{t}\left(\begin{array}{cc}
S(t-s) & 0 \\
S_{t-s} & T_{0}(t-s)
\end{array}\right)\left(\begin{array}{ll}
0 & \Phi \\
0 & 0
\end{array}\right)\left(\begin{array}{c}
S(s) x \\
S_{s} x+T_{0}(s) f
\end{array}\right) d s \\
& =\int_{0}^{t}\left(\begin{array}{cc}
S(t-s) & 0 \\
S_{t-s} & T_{0}(t-s)
\end{array}\right)\left(\begin{array}{c}
\Phi\left(S_{s} x+T_{0}(s) f\right) \\
0
\end{array}\right) d s \\
& =\int_{0}^{t}\left(\begin{array}{c}
S(t-s) \Phi\left(S_{s} x+T_{0}(s) f\right) \\
S_{t-s} \Phi\left(S_{s} x+T_{0}(s) f\right)
\end{array}\right) d s .
\end{aligned}
$$

We will prove norm continuity of both components separately. 
946 SMT for semigroups solving PDEs with nonautonomous past

(1) Let $t \geq 0,1>h>0$. Then

$$
\begin{aligned}
\left\|\int_{0}^{t+h} S(t+h-s) \Phi\left(S_{s} x+T_{0}(s) f\right) d s-\int_{0}^{t} S(t-s) \Phi\left(S_{s} x+T_{0}(s) f\right) d s\right\| \\
=\| \int_{0}^{t} S(t+h-s) \Phi\left(S_{s} x+T_{0}(s) f\right) d s \\
\quad+\int_{t}^{t+h} S(t+h-s) \Phi\left(S_{s} x+T_{0}(s) f\right) d s-\int_{0}^{t} S(t-s) \Phi\left(S_{s} x+T_{0}(s) f\right) d s \| \\
\leq \int_{0}^{t}\|S(t+h-s)-S(t-s)\|\left\|\Phi\left(S_{s} x+T_{0}(s) f\right)\right\| d s \\
\quad+\int_{t}^{t+h}\|S(t+h-s)\|\left\|\Phi\left(S_{s} x+T_{0}(s) f\right)\right\| d s .
\end{aligned}
$$

By the change of variable $s-t=: \tau$, we obtain that the last two lines in (3.25) are equal to

$$
\begin{aligned}
\int_{0}^{t}\|S(t+h-s)-S(t-s)\|\left\|\Phi\left(S_{s} x+T_{0}(s) f\right)\right\| d s \\
\quad+\int_{0}^{h}\|S(h-\tau)\|\left\|\Phi\left(S_{\tau+t} x+T_{0}(\tau+t) f\right)\right\| d \tau \\
\leq \int_{0}^{t}\|S(t+h-s)-S(t-s)\|\left\|\Phi\left(S_{s} x+T_{0}(s) f\right)\right\| d s \\
\quad+\sup _{0 \leq r \leq 1}\|S(r)\| q(h)\left\|\left(\begin{array}{l}
x \\
f
\end{array}\right)\right\| .
\end{aligned}
$$

Since the delay operator $\Phi$ satisfies condition (2.22), using the Lebesgue dominated convergence theorem and the immediate norm continuity of $(S(t))_{t \geq 0}$, we have that

$$
\int_{0}^{t}\|S(t+h-s)-S(t-s)\|\left\|\Phi\left(S_{s} x+T_{0}(s) f\right)\right\| d s+\sup _{0 \leq r \leq 1}\|S(r)\| q(h)\left\|\left(\begin{array}{l}
x \\
f
\end{array}\right)\right\|
$$

goes to zero as $h \rightarrow 0^{+}$uniformly for $\left(\begin{array}{l}x \\ f\end{array}\right) \in D\left(\mathscr{C}_{0}\right),\left\|\left(\begin{array}{l}x \\ f\end{array}\right)\right\| \leq 1$.

For $h \rightarrow 0^{-}$, the proof is analogous. Since $D(B)$ is dense in $\mathscr{E}$, it follows that the first component of $\mathscr{T}_{1}(t)$ is immediately norm continuous.

(2) For the second component we can proceed in a similar way using the norm continuity of the function $t \mapsto S_{t}$, proved in Lemma 3.5.

Hence, the map $t \mapsto \mathscr{T}_{1}(t)$ is norm continuous. 
Proposition 3.11. Under Assumption 3.2, the critical spectra of the perturbed semigroup $(\mathscr{T}(t))_{t \geq 0}$ and of the unperturbed semigroup $\left(\mathscr{T}_{0}(t)\right)_{t \geq 0}$ coincide, that is,

$$
\sigma_{\text {crit }}(\mathscr{T}(t))=\sigma_{\text {crit }}\left(\mathscr{T}_{0}(t)\right)
$$

Proof. Let $R_{k}(t):=\sum_{j=k}^{\infty} \mathscr{T}_{j}(t)$. By Proposition 3.10, the function $t \mapsto \mathscr{T}_{1}(t)$ is norm continuous. Hence, by [6, Proposition 4.7], the map

$$
t \longmapsto R_{1}(t)
$$

is norm continuous.

We are now ready to prove the spectral mapping theorem for the semigroup $(\mathscr{T}(t))_{t \geq 0}$.

THEOREM 3.12. If B generates an immediately norm continuous semigroup and $\Phi$ is as in (2.13), then

$$
\sigma(\mathscr{T}(t)) \backslash\{0\}=e^{t \sigma(\mathscr{C})} \cup \sigma\left(T_{0}(t)\right) \backslash\{0\}
$$

for $t>0$.

Proof. By Proposition 3.11, we have (3.28). Thus, applying [6, Corollary 4.6], one has

$$
\sigma(\mathscr{T}(t)) \backslash\{0\}=e^{t \sigma(\mathscr{C})} \cup \sigma_{\text {crit }}\left(\mathscr{T}_{0}(t)\right) \backslash\{0\}
$$

By Theorem 3.8 and Corollary 3.9, we know that

$$
\sigma_{\text {crit }}\left(\mathscr{T}_{0}(t)\right)=\sigma\left(T_{0}(t)\right)
$$

for $t>0$, thus the assertion follows.

The right-hand side of (3.30) determines $\sigma(\mathscr{T}(t))$ in a very satisfactory way. Indeed, $\sigma(\mathscr{C})$ can be calculated via Lemma 3.1, while

$$
\sigma\left(T_{0}(t)\right)=\left\{\lambda \in \mathbb{C}:|\lambda| \leq e^{t \omega_{0}(U)}\right\}
$$

holds by Theorem 2.10 .

\section{Application to stability}

Since the solutions $u(t)$ of $(1.1)$ are related to the semigroup $(\mathscr{T}(t))_{t \geq 0}$ (see Theorem 2.3), in order to study the stability of $u(t)$ we have to analyze the stability of $(\mathscr{T}(t))_{t \geq 0}$. For this reason we are interested in the growth bound $\omega_{0}(\mathscr{T}(\cdot))$ of the semigroup $(\mathscr{T}(t))_{t \geq 0}$. In [15, Proposition 4.3], Nagel and Poland proved that

$$
\omega_{0}(\mathscr{T}(\cdot))=\max \left\{s(\mathscr{C}), \omega_{\text {crit }}(\mathscr{T}(\cdot))\right\}
$$


By Theorem 3.8, Corollary 3.9, and Proposition 3.11,

$$
\omega_{\text {crit }}(\mathscr{T}(\cdot))=\omega_{0}(\mathcal{U})
$$

hence we obtain the following results.

TheOREM 4.1. The growth bound of $\mathcal{T}$ is given by

$$
\omega_{0}(\mathscr{T}(\cdot))=\max \left\{s(\mathscr{C}), \omega_{0}(\mathscr{U})\right\} .
$$

Corollary 4.2. If $(U(t, s))_{t \leq s \leq 0}$ is exponentially stable and there exists $\epsilon>0$ such that $\lambda \in \sigma\left(B+\Phi \epsilon_{\lambda}\right)$ implies $\Re \lambda<-\epsilon$, then the solutions of (1.1) are exponentially stable.

However, the determination of all $\lambda \in \sigma\left(B+\Phi \epsilon_{\lambda}\right)$ remains a difficult task. In this case the positivity is helpful as we can see in the rest of this section (compare [5, Example 5]).

Example 4.3. Let $X$ be the Hilbert space $L^{2}[0,1], E:=L^{p}\left(\mathbb{R}_{-}, X\right)$, and $B$ the Dirichlet Laplacian, that is, $B f:=\Delta f$ with domain $D(B):=\left\{f \in H^{2}[0,1]: f(0)\right.$ $=f(1)=0\}$. This operator generates a positive analytic semigroup $\left(e^{t \Delta}\right)_{t \geq 0}$ with $\omega_{\text {crit }}\left(e^{t \Delta}\right)=-\infty$. As in [5, Example 5] or [11, Example 4.7], we define the operators $A(s)$ as

$$
A(s):=a(s) B, \quad s \leq 0,
$$

where $0<a(\cdot) \in C\left(\mathbb{R}_{-}\right)$. We recall that these operators generate a backward evolution family $(U(t, s))_{t \leq s \leq 0}$ given by

$$
U(t, s)=e^{\left(\int_{t}^{s} a(\sigma) d \sigma\right) \Delta}, \quad t \leq s \leq 0 .
$$

Since

$$
\|U(t, s)\|=e^{\left(\int_{t}^{s} a(\sigma) d \sigma\right) \lambda_{0}}
$$

where $\lambda_{0}$ is the largest eigenvalue of $\Delta$, we can directly determine the growth bound of $(U(t, s))_{t \leq s \leq 0}$.

Proposition 4.4 (see [5, Example 5]). The growth bound of $(U(t, s))_{t \leq s \leq 0}$ is given by

$$
\omega_{0}(\mathcal{U})=\inf _{h>0} \sup _{s+h \leq 0}\left(\frac{1}{h} \int_{s}^{s+h} a(\sigma) d \sigma\right) \lambda_{0} .
$$

Consider the delay operator $\Phi$ given by

$$
\Phi f:=\int_{-\infty}^{0} \phi(s) f(s) d s
$$


for $f \in C_{0}\left(\mathbb{R}_{-}, X\right) \cap L^{p}\left(\mathbb{R}_{-}, X\right)$, where $0 \leq \phi(\cdot) \in L^{1}\left(\mathbb{R}_{-}\right)$. By Theorems 2.3 and 2.6, the delay equation (1.1) is well posed. Using the positivity of $\left(e^{t \Delta}\right)_{t \geq 0}$ and $\Phi$, we can prove, as in [5], the following result.

Proposition 4.5. The spectral bound $s(\mathscr{C})$ of the generator $\mathscr{C}$ is the unique solution of the equation

$$
\lambda_{0}+\int_{-\infty}^{0} \phi(s) e^{\lambda s} e^{\left(\int_{s}^{0} a(\sigma) d \sigma\right) \lambda_{0}} d s=\lambda
$$

Proof. By definition, we have

$$
B+\Phi \epsilon_{\lambda}=\Delta+\int_{-\infty}^{0} \phi(s) e^{\lambda s} e^{\left(\int_{s}^{0} a(\sigma) d \sigma\right) \Delta} d s
$$

Using the spectral theorem for selfadjoint operators, this implies

$$
s\left(B+\Phi \epsilon_{\lambda}\right)=\lambda_{0}+\int_{-\infty}^{0} \phi(s) e^{\lambda s} e^{\left(\int_{s}^{0} a(\sigma) d \sigma\right) \lambda_{0}} d s,
$$

where $\lambda_{0}$ is the largest eigenvalue of $\Delta$.

Since the function

$$
\lambda \longmapsto \lambda_{0}+\int_{-\infty}^{0} \phi(s) e^{\lambda s} e^{\left(\int_{s}^{0} a(\sigma) d \sigma\right) \lambda_{0}} d s
$$

is continuous and strictly decreasing, the spectral bound $s(\mathscr{C})$ is the unique solution of the equation

$$
\lambda_{0}+\int_{-\infty}^{0} \phi(s) e^{\lambda s} e^{\left(\int_{s}^{0} a(\sigma) d \sigma\right) \lambda_{0}} d s=\lambda
$$

In particular, one shows, as in [8, Theorem VI.6.14], that

$$
s(\mathscr{C})<0 \Longleftrightarrow \lambda_{0}+\int_{-\infty}^{0} \phi(s) e^{\left(\int_{s}^{0} a(\sigma) d \sigma\right) \lambda_{0}} d s<0 .
$$

Hence, by Theorem 4.1, we obtain that the semigroup $(\mathscr{T}(t))_{t \geq 0}$ is uniformly exponentially stable if and only if the second inequality of (4.14) holds and $\omega_{0}(थ)<0$.

Remark 4.6. Since the eigenvalue $\lambda_{0}$ is negative and the function $a(\cdot)$ is continuous, the above negative condition $\omega_{0}(\mathcal{U})<0$ is equivalent to the condition $\inf _{s} a(s)>0$. Thus the semigroup $(\mathscr{T}(t))_{t \geq 0}$ is uniformly exponentially stable if and only if the second inequality of (4.14) holds and $\inf _{s} a(s)>0$. 


\section{Acknowledgments}

The author is supported by the Italian Programma di Ricerca di Rilevante interesse Nazionale "Analisi e controllo di equazioni di evoluzione nonlineari" (cofin 2000). Part of this work has been written while the author was a Ph.D. student at the Department of Mathematics at the University of Tübingen and she takes the opportunity to thank Rainer Nagel for many helpful discussions.

\section{References}

[1] W. Arendt, C. J. K. Batty, M. Hieber, and F. Neubrander, Vector-Valued Laplace Transforms and Cauchy Problems, Monographs in Mathematics, vol. 96, Birkhäuser Verlag, Basel, 2001.

[2] B. Aulbach and N. Van Minh, Bounded and almost periodic solutions and evolution semigroups associated with nonautonomous functional differential equations, Abstr. Appl. Anal. 5 (2000), no. 4, 245-263.

[3] A. Bátkai and S. Piazzera, Delay equations on $L^{p}$-phase spaces, in preparation.

[4] M. Blake, S. Brendle, and R. Nagel, On the structure of the critical spectrum of strongly continuous semigroups, Evolution Equations and Their Applications in Physical and Life Sciences (Bad Herrenalb, 1998), Lecture Notes in Pure and Appl. Math., vol. 215, Dekker, New York, 2001, pp. 55-65.

[5] S. Brendle and R. Nagel, PFDE with nonautonomous past, Discrete Contin. Dynam. Systems 8 (2002), no. 4, 953-966.

[6] S. Brendle, R. Nagel, and J. Poland, On the spectral mapping theorem for perturbed strongly continuous semigroups, Arch. Math. (Basel) 74 (2000), no. 5, 365-378.

[7] C. Chicone and Y. Latushkin, Evolution Semigroups in Dynamical Systems and Differential Equations, Mathematical Surveys and Monographs, vol. 70, American Mathematical Society, Rhode Island, 1999.

[8] K.-J. Engel and R. Nagel, One-Parameter Semigroups for Linear Evolution Equations, Graduate Texts in Mathematics, vol. 194, Springer-Verlag, New York, 2000.

[9] H. O. Fattorini, Infinite-Dimensional Optimization and Control Theory, Encyclopedia of Mathematics and Its Applications, vol. 62, Cambridge University Press, Cambridge, 1999.

[10] G. Fragnelli, Semigroups and genetic repression, to appear in J. Concr. Appl. Math.

[11] Classical solutions for partial functional differential equations with nonautonomous past, Arch. Math. (Basel) 79 (2002), no. 6, 479-488.

[12] G. Fragnelli and G. Nickel, Partial functional differential equations with nonautonomous past in $L^{p}$-phase spaces, Differential Integral Equations 16 (2003), no. 3, 327-348.

[13] G. Fragnelli and L. Tonetto, A population equation with diffusion, to appear in J. Math. Anal. Appl.

[14] I. Miyadera, On perturbation theory for semi-groups of operators, Tohoku Math. J. (2) 18 (1966), 299-310.

[15] R. Nagel and J. Poland, The critical spectrum of a strongly continuous semigroup, Adv. Math. 152 (2000), no. 1, 120-133.

[16] G. Nickel, Evolution semigroups for nonautonomous Cauchy problems, Abstr. Appl. Anal. 2 (1997), no. 1-2, 73-95.

[17] F. Räbiger, R. Schnaubelt, A. Rhandi, and J. Voigt, Non-autonomous Miyadera perturbations, Differential Integral Equations 13 (2000), no. 1-3, 341-368. 
[18] N. Van Minh and N. T. Huy, Characterizations of dichotomies of evolution equations on the half-line, J. Math. Anal. Appl. 261 (2001), no. 1, 28-44.

[19] N. Van Minh, F. Räbiger, and R. Schnaubelt, Exponential stability, exponential expansiveness, and exponential dichotomy of evolution equations on the half-line, Integral Equations Operator Theory 32 (1998), no. 3, 332-353.

[20] J. Voigt, On the perturbation theory for strongly continuous semigroups, Math. Ann. 229 (1977), no. 2, 163-171.

Genni Fragnelli: Dipartimento di Matematica, Università degli Studi di Roma Tor Vergata, via della Ricerca Scientifica, 00133 Roma, Italy

E-mail address: fragnell@mat.uniroma2.it 


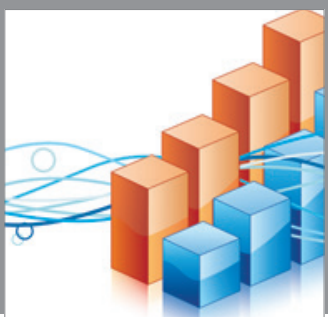

Advances in

Operations Research

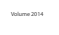

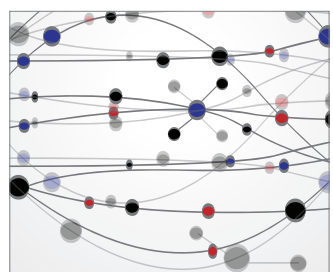

\section{The Scientific} World Journal
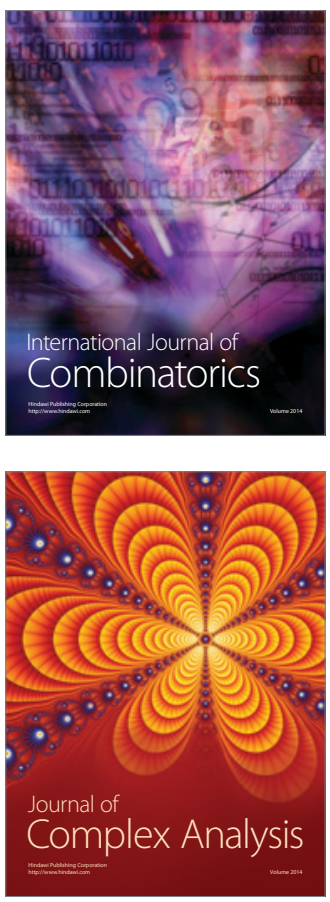

International Journal of

Mathematics and

Mathematical

Sciences
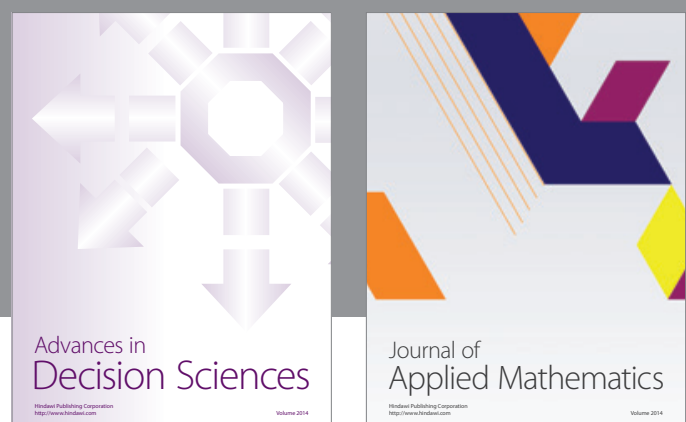

Journal of

Applied Mathematics
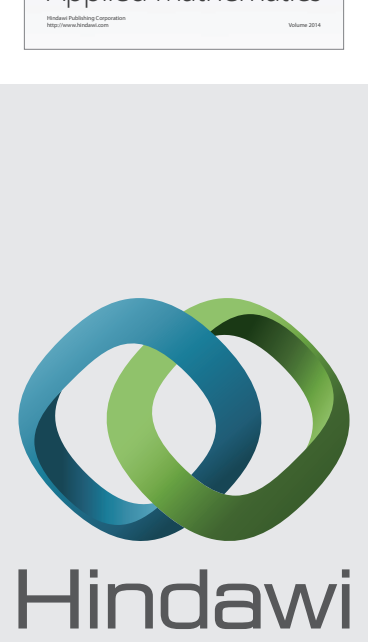

Submit your manuscripts at http://www.hindawi.com
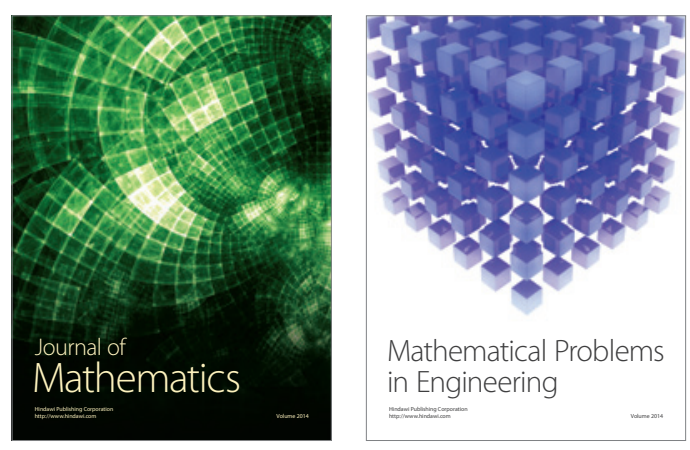

Mathematical Problems in Engineering
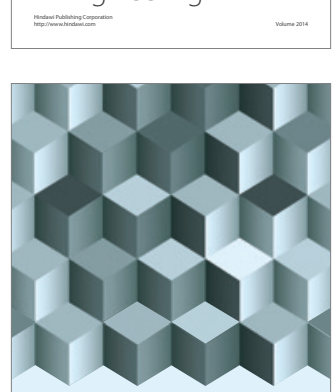

Journal of

Function Spaces
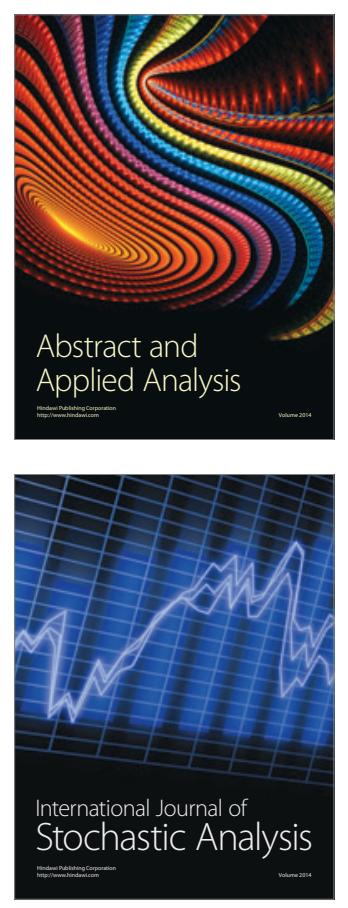

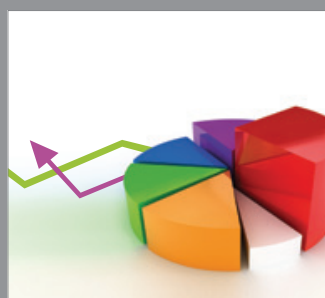

ournal of

Probability and Statistics

Promensencen
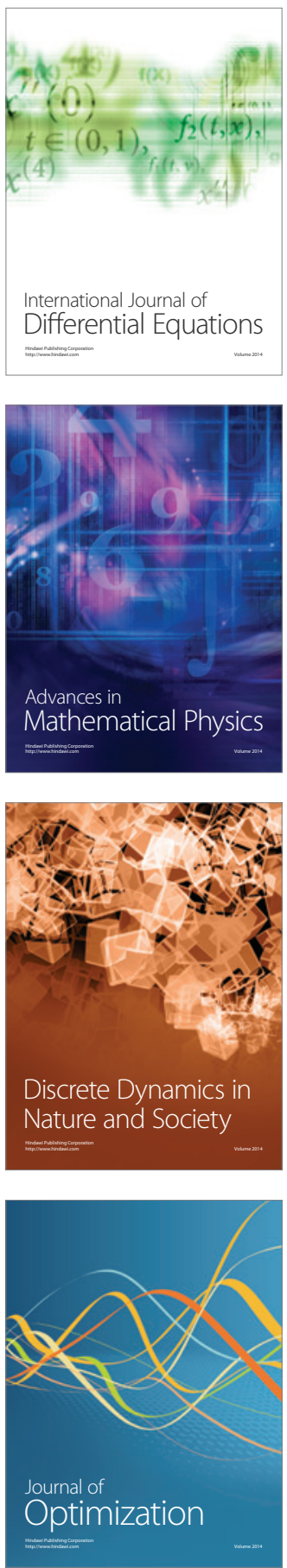